\title{
Historia y epidemiología del meningococo
}

\author{
JAN WILHELM B. ${ }^{1}$, RODOLFO VILLENA M. ${ }^{2}$ \\ 1. Pediatra Infectologo, Jefe Unidad Infectología Pediatrica, Clínica Alemana - Universidad del Desarrollo. \\ 2 Pediatra Infectologo, Jefe Unidad Infectología, Hospital Exequiel González Cortéz, Universidad de Chile.
}

\begin{abstract}
Meningococcal disease history and epidemiology

The meningococcus or Neisseria meningitidis is a bacterium that lives exclusively in the pharynx of some people, which occasionally can cause diseases such as meningitis, pneumonia, arthritis and sepsis. There are several strains of Neisseria meningitidis, like A, B, C, Y and W-135 serogroups. Historically, group C strain had been the most common in Chile, but the B group became subsequently dominant. Over the past six months, we have seen an increase in cases of meningococcal disease caused by serogroup W-135, mainly in the Santiago Metropolitan Region, as well as an increased number of B Meningococcal cases in Valparaiso. As the meningococcus epidemiology is unpredictable and dynamic, with short-term changes and outbreaks every 8 to 12 years, it requires a systematic and strict close observation in each country. The aim of this article is to review the history and epidemiology of meningococcal disease in light of current medical knowledge. To address the increasing number of cases, it is necessary to educate health teams and people about strategies to diagnose and treat this disease as early as possible, including effective preventive measures and decisionmaking regarding the use of specific vaccines.
\end{abstract}

(Key words: Neisseria meningitidis, serotype W-135, Epidemiology).

Rev Chil Pediatr 2012; 83 (6): 533-539

\section{RESUMEN}

El meningococo o Neisseria meningitidis es una bacteria que vive exclusivamente en la faringe de algunas personas y ocasionalmente puede producir enfermedades como meningitis, neumonía, artritis y sepsis. Existen varias cepas de Neisseria meningitidis, destacando los serogrupos A, B, C, Y, y W-135. Históricamente la cepa $\mathrm{C}$ era la habitual en Chile, pero posteriormente la B pasó a ser la predominante. Durante los últimos 6 meses hemos observado un aumento de los casos de infección por el serogrupo W135, fundamentalmente en la Región metropolitana, y un aumento de los casos en Valparaíso asociado a Meningococo B. Considerando que la epidemiología del meningococo es impredecible, dinámica, con cambios en corto tiempo y brotes cada 8 a 12 años, requiere de una vigilancia sistemática y estricta en cada país. El objetivo del presente artículo es revisar la historia y epidemiología del meningococo. Frente al aumento de los casos es necesario educar a los equipos de salud y a la población en estrategias tendientes al diagnóstico y tratamiento lo más precoz posible e implementación de medidas de profilaxis efectiva, además de la toma de decisiones respecto de la incorporación de vacunas específicas.

(Palabras clave: Neisseria meningitidis, serotipo W-135, epidemiología).

Rev Chil Pediatr 2012; 83 (6): 533-539

Conflicto de intereses: El Dr. Villena se desempeña como asesor médico para Novartis Vacunas Chile.

Recibido el 5 de noviembre de 2012, aceptado para publicación el 25 de noviembre de 2012 .

Correspondencia a:

Jan Wilhelm B.

janpaulwilhelm@gamil.com 


\section{Historia}

La primera vez que se describe la "meningitis epidémica" es en el año 1805, cuando el Dr. Gaspard Vieusseux describe el cuadro clínico de meningitis durante una epidemia, en Ginebra, Suiza. El microorganismo causal logra ser cultivado e identificado en 1887 por el Dr. Anton Weichselbaum, en Viena. Weichselbaum identifica diplococos gram negativos, con forma de "granos de café", al interior de piocitos obtenidos desde el líquido céfaloraquídeo (LCR) de un paciente con meningitis, bautizándolos como Diplococcus intracellularis meningitidis. Posteriormente se descubrirá que la bacteria puede encontrarse en la faringe de personas sanas y que existen distintos tipos de meningococo. El microorganismo será reclasificado como Neisseria meningitidis.

En 1911 el Dr. Rupert Waterhouse, y posteriormente el Dr. Carl Friderichsen en una revisión más amplia, describe la necrosis hemorrágica de las glándulas suprarrenales en el curso de una enfermedad infecciosa grave. Neisseria meningitidis es el agente etiológico más frecuentemente involucrado en el desarrollo de este síndrome, aunque otros agentes pueden también provocarlo. En la primera guerra mundial se observa que el porcentaje de reclutas "portadores" de la bacteria aumenta significativamente en períodos de mayor hacinamiento.

Entre 1928 y 1945 se produjeron numerosas epidemias (Detroit 1928-1929, Milwaukee 1927-1929, Chile 1941-1943), con tasas de letalidad que alcanzan el 50\%. A comienzo de los años 30 un médico alemán, Gerhard Domagk, descubre las sulfas. Hacia el final de esa década se publican los primeros resultados del tratamiento de la meningitis aguda con sulfonamidas, intervención que logra disminuir la letalidad a 15\%. Además, las sulfonamidas comienzan a ser utilizadas con éxito para prevenir la enfermedad en contactos de personas con meningitis.

Lamentablemente, en menos de 5 años surge resistencia a sulfonamidas. En 1943 el Dr. Alexander Fleming, inglés, publica el descubrimiento de la penicilina, por el cual recibe en 1943 el premio Nobel. Sin embargo, pron- tamente se constata que los antibióticos (sulfonamidas o penicilina) no son suficientes para controlar el problema en la población, con lo cual surge una nueva estrategia: el desarrollo de vacunas contra el meningococo. Habrá que esperar hasta 1970 para que se inicie exitosamente el uso de la primera vacuna contra Neisseria meningitidis serogrupo $\mathrm{C}$.

\section{Agente etiológico}

Neisseria meningitidis es un diplococo gram negativo, aerobio, inmóvil. El genus Neisseria también incluye a $N$. gonorrhoeae y a otros comensales, como $N$. flavescens, $N$. lactamica, N. mucosa, N. sicca y N. subflava. Su envoltura celular se compone de tres elementos, una membrana externa con proteínas, lipooligosacáridos y fosfolípidos, una membrana citoplasmática y una pared celular de peptidoglicanos (figura 1). Las formas invasoras poseen además una cápsula y pilis o fimbrias. El meningococo posee complejos requerimientos nutricionales para subsistir, por lo que es altamente susceptible al calor, el frío, luz solar y $\mathrm{pH}$ adverso. La cápsula polisacárida permite su división en 13 serogrupos designados por las letras: A, B, C, D, X, Y, Z, 29 E, W 135, H, I, K y L. Los serogrupos que se relacionan con enfermedad en seres humanos son A, B, C, Y y W-135, con

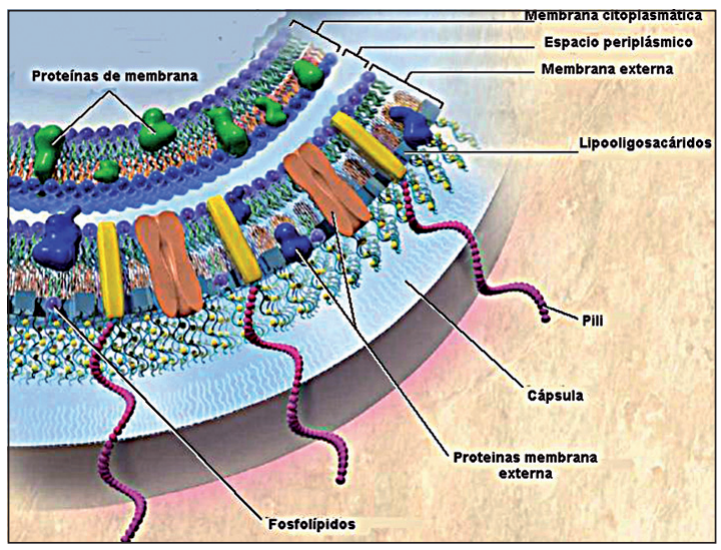

Figura 1. Estructura de la envoltura de Neisseria meningitidis. Reproducida de la publicación original D. S. Stephens, B. Greenwood, and P. Brandtzaeg. Epidemic meningitis, meningococcaemia, and Neisseria meningitidis. Lancet 369 (9580): 2196-2210, 2007. 
recientes descripciones del serogrupo $\mathrm{X}$. Las proteínas de la membrana externa Por A y Por B definen los 20 serotipos/subserotipos conocidos. Los inmunotipos se clasifican según la estructura de los lipooligosacáridos y las cepas según análisis genético utilizando multi-locus sequence typing (MLST), lo que nos ha permitido conocer a las cepas hipervirulentas. El genoma meningocócico contiene varias secuencias de inserción que le permiten obtener ADN del ambiente microbiano, lo que ha utilizado para adquirir operones capsulares e intercambiarlos, por ejemplo entre serogrupos $\mathrm{B}$ y $\mathrm{C}$.

La cápsula polisacárida le permite evadir fagocitosis, sobrevivir a la desecación e invadir a través de la mucosa nasofaríngea a los sujetos colonizados. La colonización precede a la infección, sin embargo, no se conoce exactamente cuáles son los eventos que gatillarían que esto ocurra. Durante la invasión al torrente sanguíneo se libera endotoxina (LPS) que desencadena una cascada proinflamatoria, lo que lleva a los pacientes a desarrollar shock séptico en los casos más severos y con mayor concentración plasmática de LPS.

Otro evento relacionado con la susceptibilidad a la infección meningocócica es la presencia de anticuerpos bactericidas séricos en la población, los cuales serían mayores en adultos que en niños, lo que explicaría la mayor incidencia en estos últimos y su escasez en menores de 6 meses de edad. Esta respuesta bactericida requiere de anticuerpos y complemento para que sea funcional, por lo tanto aquellos pacientes con dichas falencias estarían en mayor riesgo para padecer de una infección meningocócica invasora. La portación de $N$. lactamica o de otras $N$. meningitidis no tipificables podrían inducir actividad bactericida cruzada contra los serogrupos invasores.

La nasofaringe humana es el único reservorio natural conocido. La transmisión ocurre a través de gotitas, por exposición directa y prolongada a un portador asintomático o a un enfermo. Habitualmente existe portación nasofaríngea que oscila, durante periodos no epidémicos, entre un 1 a un $15 \%$ de la población, variando ampliamente según el grupo etario, con predominio entre adolescentes y adultos jóvenes y en niveles socioeconómicos bajos. La portación aumenta durante los períodos epidémicos hasta un $30-40 \%$, pero no se correlaciona con desarrollo de brotes ni se conoce su duración. El mayor riesgo de desarrollar enfermedad ocurre durante los 10 días posteriores al contacto, siendo 500 a 800 veces mayor al de la población general, lo que da las bases para el uso de quimioprofilaxis en los contactos. La transmisión persiste hasta que los meningococos desaparecen de la nariz y de la boca, habitualmente a las 24 horas de quimioprofilaxis o tratamiento antibiótico. La penicilina sódica no erradica, por lo que se requiere utilizar alguno de los fármacos sugeridos en la profilaxis para lograr este objetivo (por ejemplo, cefalosporinas de $3^{\circ}$ generación, rifampicina, azitromicina y/o ciprofloxacino). El período de incubación no se ha precisado, pero se encuentra entre los 2 y 10 días, siendo habitual 3 a 4 días.

\section{Cuadro clínico}

La enfermedad meningocócica invasora (EMI) tiene implicancias individuales y comunitarias, pudiendo presentarse de distintas formas, independiente del estado de salud de los sujetos, desde un síndrome febril sin foco con buen estado general, hasta un cuadro de shock séptico fulminante (figura 2), pasando por meningitis, sepsis, neumonía, artritis y pericar-

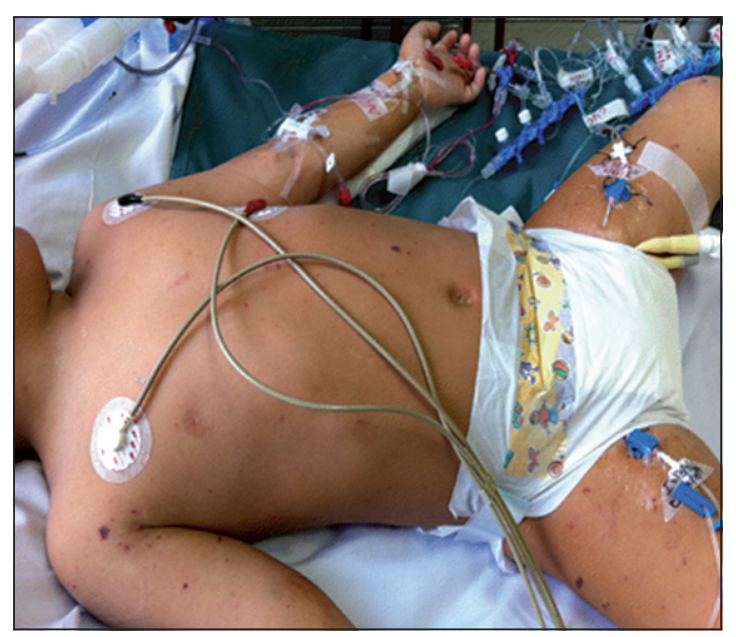

Figura 2. Foto meningococcemia. Dr. Rodolfo Villena. 
ditis. La letalidad general de la enfermedad meningocócica invasora es de 8-14\%, y del $5-15 \%$ cuando se presenta como meningitis. Esta letalidad no ha variado desde 1939, año en que se introdujo la terapia con sulfas. Las secuelas a largo plazo en los sobrevivientes alcanzan el 11-19\%, manifestándose principalmente como amputaciones, sordera y discapacidades neurológicas. Sin embargo, un estudio reciente en el Reino Unido demuestra que si se incluyen trastornos psicólogicos, la cifra de secuelas aumenta hasta el $36 \%$. Las manifestaciones iniciales son inespecíficas, similares a las de un cuadro gripal, pudiendo progresar rápidamente a sepsis en algunos casos, lo que se hace más evidente en los menores de cuatro años. El exantema meningocócico es tardío dentro de la evolución de la enfermedad, por lo que su detección debe ser indicador de urgencia en el inicio del manejo del paciente.

El diagnóstico es clínico, con confirmación microbiológica desde sitios estériles (sangre, LCR, líquido articular) o lesiones cutáneas. Debe considerarse una urgencia antibiótica, con administración de antibióticos bactericidas durante la reanimación del paciente, previa toma de cultivos, pero estos no deben retrasar el manejo general del paciente. El manejo es hospitalizado, idealmente en una unidad de paciente crítico, con precauciones de gotitas $\mathrm{y}$ antibióticos endovenosos. Actualmente en Chile la sensibilidad a PNC es de $51 \%$, y de $100 \%$ para ceftriaxona, por lo que esta última sería la de indicación para el manejo inicial. Todo caso sospechoso debe ser notificado de manera inmediata para detectar aparición de brotes y realizar el manejo de contactos, en caso que se requiera.

Los factores de riesgo descritos como asociados al desarrollo de EMI son sistema inmune inmaduro o alterado (inmunodeficiencia humoral, déficit de componentes terminales del complemento C5-C9, déficit de properdina), irritación nasofaríngea, tabaquismo, hacinamiento, contacto estrecho con un caso, viajeros/residentes en zonas endémicas y falta de anticuerpos bactericidas contra la cepa infectante, lo que podría explicar que la mayoría de los casos ocurren en personas previamente sanas $\sin$ factores de riesgo identificados.

\section{Epidemiología}

La distribución de $N$ meningitidis es endémica a nivel mundial, describiéndose casos en todos los continentes y epidemias en todos los países, independiente de su nivel de desarrollo. La EMI es de baja incidencia, pero de muy alta letalidad, superior al $10 \%$ de los casos, afectando principalmente a personas sanas. El patrón de ocurrencia endémico, con tasas por cien mil habitantes, en países desarrollados es de 0.28 a 5 casos, con mayor incidencia en niños entre 3 meses a 5 años y predominio de serogrupo B. En tanto en los países en desarrollo, como el Africa sub-Sahariana presenta tasas de 10 a 20 casos/100 000 hab., con predominio del serogrupo A, en niños de 5-10 años. El patrón de ocurrencia epidémico tiene su máxima expresión en el "cinturón de meningitis africano", con tasas de hasta 1000 casos por 100000 hab, con predominio de serogrupo A. En América los serogrupos predominantes durante las epidemias son el B y C, y los serogrupos endémicos corresponden a predominio de W 135 en Argentina, de C en Brasil y de B en Chile. Las tasas latinoamericanas van desde $0,05 / 100000$ hab. en México hasta 1,8/100 000 hab en Brasil. Sin embargo, los reportes son inconstantes y se sospecha de falencias en los sistemas de vigilancia. En los últimos años el serogrupo $\mathrm{W}$ 135 ha emergido como una causa frecuente desplazando incluso al B en algunos países. La introducción de vacunas conjugadas contra el serogrupo C modificó la epidemiología en los países donde se implementó, pero sin producir un reemplazo en los serogrupos respecto de EMI.

La epidemiología del meningococo, por lo tanto, es impredecible, dinámica, con cambios en corto tiempo y brotes cada 8 a 12 años, habitualmente en invierno y primavera, requiriendo una vigilancia sistemática y estricta para tomar decisiones adecuadas respecto de vacunación. En Chile su vigilancia a través del Instituto de Salud Pública se inició en 1976, con análisis y referencia de todas las cepas identificadas desde muestras estériles. Nuestra historia no ha estado ajena a los brotes por meningococo, contándose el de 1942 de carácter nacional, por el serogrupo A, con variadas publicaciones al respecto, el de 1978 en la Región Metro- 
politana por el serogrupo C, 1979 en Osorno, nuevamente por serogrupo A, 1980 y 1993 en Iquique y Región Metropolitana por serogrupo B y el año 2000 en Concepción, Aysén, Magallanes por serogrupo $\mathrm{C}$ (figura 3 ).

\section{Situación actual}

Hasta el 01/10/2012 se han producido 83 casos de enfermedad meningocócica en el país (la mediana de los últimos 5 años es de 74 casos) Esto da como resultado una tasa para Valparaíso de 0,9 casos por 100000 habitantes y para Santiago de 0,7 casos por 100000 hab. Hasta la fecha no se ha establecido algún nexo epidemiológico en las zonas más afectadas, sin embargo, se han confirmado 48 casos de Enfermedad Meningocócica por Serogrupo W-135, cifra que duplica el total de casos por W135 observado durante todo el año 2011 (figura 4). La Región Metropolitana concentra el 81\% (39 casos) del total de casos por serogrupo $\mathrm{W}-135$, lo que da una tasa de incidencia serotipo específica de 0,6 por cien mil habitantes, lo que duplica a la tasa del país $(0,3$ por cien mil habitantes).

Con relación a las características de los casos, el rango de edad es de 3 meses a 93 años. Del total de casos, 24 se encuentran en el grupo de menores de 5 años; y de éstos, 10 son menores de 9 meses. Hasta la semana 46, se han reportado 10 defunciones por este serogrupo, lo que representa una tasa de letalidad de $21 \%$, cifra superior a la esperada para la enfermedad (10$15 \%$ ) (figura 5). El rango de edad de los fallecidos es de 6 meses a 93 años y los hombres representan $70 \%$.

\section{Discusión}

Durante los últimos 6 meses hemos observado un aumento de los

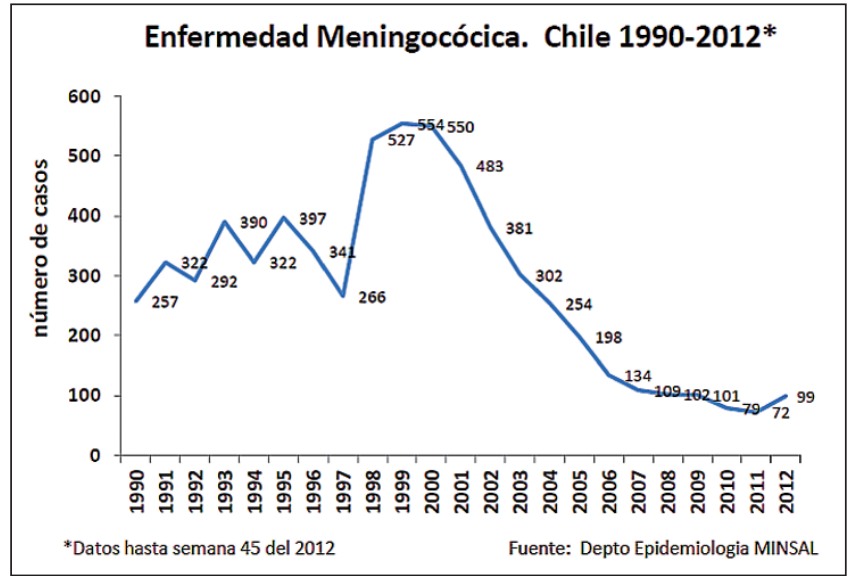

Figura 3. Número de casos enfermedad Meningocócica. Chile 19902012. Fuente: Depto. Epidemiologia MINSAL.

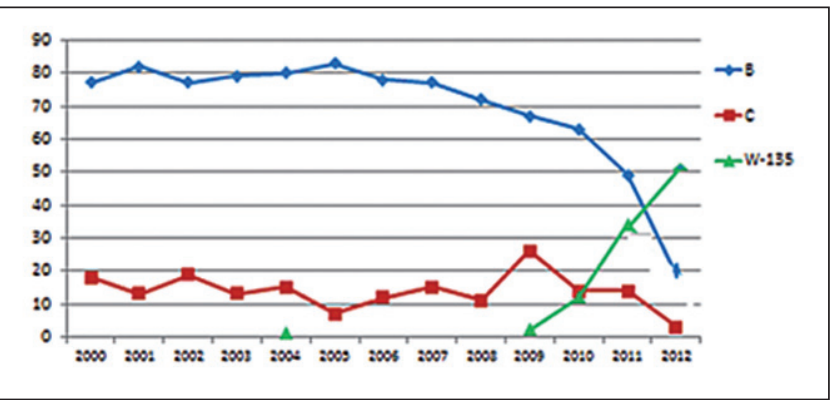

Figura 4. Casos enfermedad Meningocócica por serogrupo. Chile 2000-2012 (SE 46). Fuente: Depto. Epidemiologia MINSAL. Gentileza Dra. Cecilit.

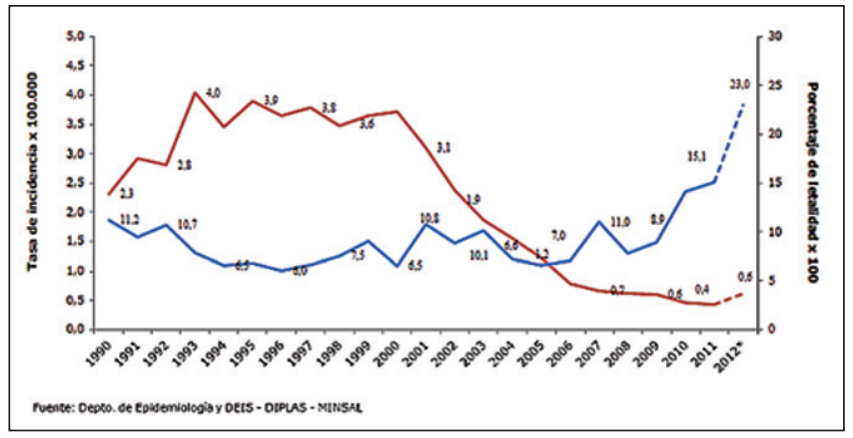

Figura 5. Tasa de incidencia y porcentaje de letalidad enfermedad Meningocócica. Chile 1990-2012(SE 46). Fuente: Depto Epidemiologia MINSAL.

casos de infección meningocócica por el serogrupo W135, fundamentalmente en la Región Metropolitana, $\mathrm{y}$ un aumento de los casos en Valparaíso asociado a Meningococo B.

Actualmente la tasa para la enfermedad menin- 
gocócica en el país es de 0,5/100 000 habitantes. Valparaíso presenta una tasa de 0,9 casos por 100000 habitantes y Santiago una tasa de 0,7 casos por 100000 habitantes, muy por debajo de lo establecido por la definición de brote del CDC (10/100 000 habitantes) Incluso cuando se hace un análisis por comuna, las tasa de notificación más altas alcanzan 2/100 000 habitantes. Por otra parte, es probable que el que el número de casos y la proporción de cuadros clínicos causados por W-135 siga aumentando, dado el cambio endémico que se está produciendo.

Con el objetivo de prevenir la aparición de nuevo casos, particularmente de personas susceptibles, el Ministerio de Salud implementó una estrategia de vacunación para los niños entre 9 meses y 5 años de todo el país. Antes del invierno del 2013 todos los niños del país, dentro del grupo objetivo, deberán estar vacunados.

Lo anterior hace plantearse ¿cuál es el objetivo de usar una vacuna? En estrategias en inmunizaciones las alternativas son 4:

1) Erradicar una enfermedad: Esta es la excepción más que la regla. Es una estrategia costosa y de difícil implementación. A nivel mundial la viruela, y tal vez pronto la poliomielitis, es el único ejemplo.

2) Controlar un brote: Como se hace en brotes por virus hepatitis A. En el caso de meningococo el control de brote se realiza con quimioprofilaxis antibiótica, conociéndose una sola experiencia de uso de vacuna (control de casos secundarios durante brote de meningococo en peregrinación a la Mecca) con resultados inciertos.

3) Desarrollar inmunidad en la población (inmunidad de rebaño): El ejemplo clásico es el Sarampión, donde se logra proteger a suceptibles bloqueando la transmisión del microorganismo. Requiere tasa de cobertura alta en la población, generalmente mayor al $80 \%$.

4) Desarrollar inmunidad en el individuo suceptible para protegerlo: Este es el caso de la vacunación anual contra la influenza y es la estrategia que adoptó el MINSAL en el caso de meningococo W-135.
Las vacunas disponibles demuestran alta seroconversión, con niveles seroprotectores contra la cepa W135, lo que protegerá a la población en riesgo, pero no se puede esperar que la inmunización erradique la enfermedad o genere inmunidad de rebaño. Por lo tanto, es probable que el serotipo W-135 haya llegado para quedarse y la pregunta de fondo es si debemos, ahora que se inició esta estrategia, incorporar la vacuna al Plan Nacional de Inmunizaciones. Todos los años habrá una nueva cohorte de chilenos suceptibles y el W-135 estará esperando. La pregunta solo puede ser resuelta con estudios de costo-efectividad para cada una de las intervenciones en las diferentes enfermedades que afectan a nuestra población.

\section{Referencias}

1.- Vacunación contra el meningococo. Manual de vacunas en pediatría 2da edición latinoamericana. Págs 190-202 Sociedad Latinoamericana de infectología pediátrica. Asociación Panamericna de Infectología. Asociación Española de Infectología.

2.- Rosenstein NE: Meningococaldisease New England Journal Med 2003; 344 (18): 1378-88.

3.- Palazzi Sáfadi MA, Leite Cintra OA: Epidemiology of meningococcal disease in Latin America: current situation and opportunities for prevention. Neurological Research 2010; 32 (3): 263-71.

4.- Granoff DM, Harrison L, Borrow R: Meningococcal vaccines. In: Plotkin SA, Orenstein WA, Offit PA, editors. Vaccines. 5th ed. Saunders; 2008.

5.- Instituto de Salud Pública de Chile: Boletín Vol. 2, No 2, marzo 2012.

6.- Organización Mundial de la Salud: Vacunas meningocócicas. WHO position paper. Reporte epidemiológico semanal. Weekly epidemiological record.Noviembre 18, 2011, año 86to. No. 47, 2011, 86, 521-40. http:// www.who.int/wer

7.- Trotter $C L$, et al: Meningococcal vaccines and herd immunity: Lessons learned from serogroup $\mathrm{C}$ conjugate vaccination programs. Expert Review of Vaccines 2009; 8: 851-61.

8.- Advisory Committee on Immunization Practices (ACIP): Licensure of a meningococcal conjugate vaccine for children aged 2 through 10 years and updated booster dose guidance for adolescents and other persons at increased risk for meningococcal disease. MMWR Morbidity and Mortality Weekly Report 2011; 60: 1018-9. 
9.- Santolaya ME, O'Ryan ML, Valenzuela MT, et al: Immunogenicity and tolerability of a multicomponent meningococcal serogroup B (4CMenB) vaccine in healthy adolescents in Chile: a phase $2 b / 3$ randomised, observer-blind, placebo-controlled study. Lancet 2012; 379: 617.

10.- Granoff DM: Review of meningococcal group B vaccines. Clin Infect Dis 2010; 50 Suppl 2: S54.

11.- Villena $R$ : Vacunas no incluídas en el Programa nacional de inmunizaciones Pediatría ambulatoria Quezada A. cap 22. Editorial mediterraneo, Chile, 2da ediciónpágs 232-53.

12.- American Academy of Pediatrics: Meningococcal infections. In: Red Book: 2009 Report of the Committee on Infectious Diseases, 28th ed, Pickering LK (Ed), American Academy of Pediatrics, Elk Grove Village, IL 2009. p. 455.

13.- Halperin $S$, et al: Comparison of the safety and immunogenicity of an investigational and a licensed quadrivalent meningococcal conjugate vaccine in children 2-10 years of age. Vaccine 2010; 28: 7865-72.

14- Greenwood B, Chiarot E, McLennan C, O'Ryan M: Can we defeat meningococal disease in low and middle income countries? Vaccine 2012; 30S: B63-6.

15.- Deasy A, Read RC: Challenges for development of meningococcal vaccines in infants and children Expert Rev Vaccines 2011; 10 (3): 335-43.

16.- Pace D, Pollard A: Meningococal disease: Clinical presentation and sequelae Vaccine 2012; 30S: B3-9.

17.- Black, Plotkin: Meningococcal disease fron the public health policy perspective Vaccine 2012; 30S: B37-9.

18.- Halperin SA, Bettinger J, Harrison L, et al: The changing and dynamic epidemiology of meningococcal disease. Vaccine 2012; 30S: B26-36.

19.- Organización Panamericana de la Salud: Informe Regional SIREVA II Reporte 2011: datos por país y por grupos de edad sobre las características de los aislamientos de Streptococcus pneumoniae, Haemophilus influenzae y Neisseria meningitidis en procesos invasores. http://new.paho.org

20.- Viner R, Booy R, Johnson H, et al: Outcomes of invasive meningococcal serogroup B disease in children and adolescents (MOSAIC): a case-control study. LancetNeurology www.thelancet.com/neurology Publicación online agosto 3, $2012 \mathrm{http} / /$ dx.doi.org/10.1016/S14744422 (12) 70180-1. 